\title{
Profiles and Development of Adaptive Behavior in Adolescents and Adults with Williams Syndrome
}

\author{
Trista Juhsin Fu
}

\begin{abstract}
Williams Syndrome (WS) is associated with deficits in adaptive behavior and an uneven adaptive profile. This study cross-sectionally examined the profiles and developmental trajectories of adaptive behavior in 100 adolescents and adults with Williams Syndrome ages 12 to 53 years using the Scales o Independent Behavior-Revised (SIB-R). Profile findings indicated a significant weakness in motor skills and community living skills relative to social interaction and communication skills and personal living skills. Age-related decline in Social Interaction and Communication domain was also found in this population. Interventions should address promoting adaptive motor functioning and community living skills while taking their developmental needs into consideration.
\end{abstract}

Index Terms-Adaptive behavior, development, profile, Williams syndrome.

\section{INTRODUCTION}

Williams Syndrome (WS) is a neurodevelopmental disorder characterized by a distinctive cognitive and personality profile [1], [2]. The cognitive phenotype of WS is characterized by relative strengths in auditory rote memory and language, and relative weakness in visuospatial perception, construction, and integration. Individuals with WS often have extreme difficulty with tasks involving visual-spatial construction such as drawing and block design (pattern construction) [3]-[5]. WS is also associated with profound motor planning deficits, which might play a role in poor visual-motor performance [6]. In addition, individuals with WS have an unusual personality profile associated with high sociability, overfriendliness, empathy and excessive anxiety [7]-[10]. The syndrome results in mild to moderate intellectual or learning disability [11].

"Adaptive behavior" refers to the attainment of developmentally appropriate milestones in skills that promote independence and help individuals cope with the demands of their everyday environment [12], [13]. Research

has demonstrated that WS is associated with deficits in adaptive behavior [14]-[16]. Researchers found that children with WS obtained significantly lower scores in adaptive behavior compared to their IQ- and chronological age (CA) matched counterparts with nonspecific intellectual disability [17]. Another study also found that, despite having a similar degree of general cognitive impairment, relatively few individuals with WS were able to attain a reasonable level of

Manuscript received June 5, 2014; revised August 12, 2014. This work was supported in part by a grant from the U.S. National Institutes of Health (PO1 HD33113) awarded to Dr. Ursula Bellugi at the Laboratory for Cognitive Neuroscience at The Salk Institute for Biological Studies.

T. J. Fu is with the National Chengchi University, Taipei City, Taiwan, R.O.C. (e-mail: jfu@nccu.edu.tw). independence, or cope with the demands of employment when compared to groups of adults with other intellectually disabling genetic disorders (i.e., Down syndrome and Prader-Willi syndrome) [18].

Furthermore, it has been reported that individuals with WS have uneven profiles in different domains of adaptive functioning, as measured by the Vineland Adaptive Behavior Scales (VABS) [19]. Overall, the adaptive profile of the children with WS is consistent with their cognitive profile in which communication and social interaction are the areas of strength whereas daily living and motor domains are the areas of weakness [20], [21]. However, for adults with WS, while they still exhibit a relative strength in Socialization, their performance in the Communication domain is relatively lower than their performance in the Daily Living domain on the VABS [15]. Additionally, their adaptive motor functioning was unknown since the motor domain on the VABS is only applicable for children under 6 years old.

Researchers have considered developmental differences in individuals with WS in association with their adaptive behavior. For example, a study showed that adults (aged 19 to 39) with WS did not appear to show increasing development in adaptive behavior, as assessed on the VABS [15]. Another study found that children (aged 4 to 8) with WS continued to gain in adaptive behavior abilities as they got older, as indicated by the positive correlation between the VABS age equivalent and CA [21]. However, there were limitations to this conclusion due to the narrow age range and the cross-sectional investigation method used in their study [21].

Elison and colleagues examined age associated changes in a number of different domains of functioning in adults with WS, including physical health, educational and occupational levels, self-care and independence, social interactions, and behavioral difficulties [22]. Ninety-two individuals with genetic confirmation of WS (50 males, 42 females) participated in a cross-sectional study (age range 19-55 years; mean 32 years) and 43 individuals ( 21 males, 22 females) were involved in a longitudinal study. Cross-sectional analyses revealed no age related differences in the areas of functioning investigated. However, in the longitudinal sample, significant improvements were reported in physical health, self-care and occupational skills. Social skills and adaptive behaviors had generally improved while behavioral difficulties had declined. These findings are consistent with prior studies in indicating no age related decline in social or adaptive functioning in adults with WS, at least up to the age of 50-55 years. Similar results were also found in a longitudinal study that assessed cognition in WS using the Woodcock Johnson Test of Cognitive Ability-Revised [23] twice over a 5 year period. Results suggested cognitive 
strengths and weaknesses in WS were consistent, at least on those abilities assessed using the WJ-R COG [24].

Developmental studies examining cognitive changes in specific genetic conditions, such as Down syndrome or Fragile $\mathrm{X}$, note a decrease in IQ scores as individuals reach adulthood [25]. However, as individuals with WS grow older, there is not much change in IQ scores obtained using the WISC-R and the WAIS-R [26], [27]. In a longitudinal study, Udwin and colleagues [27] followed 23 WS adolescents for eight to nine years. Measurements of cognitive ability showed a slight increase in Full Scale, Verbal and Performance IQ scores. Comparisons of reading, spelling and arithmetic scores revealed only modest increases in reading accuracy and spelling scores, a slight decline in reading comprehension scores, and little change in arithmetic test scores. The results suggested that individuals with WS make little progress in their educational skills beyond their early teenage years. In another study, Searcy et al. [26] found increased age was associated with increased Performance IQ (PIQ) but not Verbal IQ (VIQ). However, despite this age-related increase in PIQ, the results imply that the overall IQ of an adult with WS will likely remain stable.

Although children with WS do exhibit marked visual-spatial and visual-motor problems compared to children of the same chronological age, it has been suggested that these difficulties may diminish with age and/or experience [28]. Neuroimaging studies have also shown that there are important age-related changes in typical developing individuals in white and grey matter tracts and connections [29]. They found that the frontal areas, which are important for affective and behavior regulation, develop more slowly than the rest. Based on these findings, one would expect to find age differences in adaptive behavior due to brain maturation among WS individuals. Information on the developmental trajectory of adaptive behavior, and whether it reflects differences in cognitive and motor functioning, is likely to provide insights into the nature of the functional deficits in WS.

Few studies have examined the adaptive profile in adolescents with WS. In addition, no studies have examined the motor adaptive functioning in adolescents and adults with WS due to the age limitation with the motor domain in the VABS. The primary purpose of this study was therefore to systematically investigate the profiles of adaptive behavior in adolescents and adults with WS utilizing a different adaptive behavior scale (SIB-R) which allows us to look at the motor skills and community living skills in all age groups. The second purpose of this study was to examine age-related changes in the development of adaptive behavior. By identifying profiles and development of adaptive behavior in adolescents and adults with WS, this study may help to design age-appropriate interventions to further improve the quality of life of individuals with WS.

\section{METHODS}

\section{A. Participants}

Participants included one hundred individuals with WS (43 males, 57 females; mean age 28.23 years, $S D=10.63$, range 12 to 53 years). All the participants were identified from an existing database at the Laboratory for Cognitive Neuroscience (LCN) of the Salk Institute. Only individuals who had concurrent IQ and SIB-R scores in the database were included for evaluation in this study. The mean Full Scale IQ for participants was $64(\mathrm{SD}=10)$. The Verbal IQ and Performance IQ mean scores were $69(\mathrm{SD}=10)$ and $63(\mathrm{SD}=9)$ respectively.

\section{B. Procedures}

The Laboratory for Cognitive Neuroscience (LCN) of the Salk Institute for Biological Studies has been collecting neurocognitive and behavioral data on individuals with WS for over a decade. Participants were administered a comprehensive neuropsychological battery. Primary caretakers were also asked to complete various measures regarding their child's medical history, adaptive functioning, and personality and social-emotional functioning. All primary caretakers signed informed consent forms. Where appropriate, participants gave written assent before starting protocols. A data set of one hundred adolescents and adults with WS was generated from the database at the LCN for this study.

\section{Measures}

Adaptive behavior was measured by the Scales of Independent Behavior-Revised (SIB-R) [30], which is a questionnaire designed to measure various areas of adaptive and maladaptive behaviors from infancy through adulthood. It was normed on 2,000 individuals and has adequate reliability and validity [30]. It can be administered in a structured interview or by a checklist procedure. In the current study, participants' parents or caregivers completed a SIB-R checklist to provide data on participants' functional independence and adaptive behavior in home, social, school, work, and community settings. It is comprised of 14 subscales organized into four adaptive behavior clusters: Social Interaction and Communication Skills, Personal Living Skills, Community Living Skills, and Motor Skills. The Motor Skills subscales examine gross motor (e.g., rides bicycle, pours liquid from glass/pitcher) and fine motor abilities (e.g., prints first name, fastens clothing). The Social Interaction and Communication Skills subscales examine social interaction (e.g., waits for turn in group activity, takes part in games or activities), language comprehension (e.g., follows two-part directions, responds to common signs and symbols), and language expression (e.g., says last name when asked, uses complex sentences). The Personal Living Skills subscales examine eating and meal preparation (e.g., takes appropriate portions, prepares simple snacks), toileting (e.g., controls bowel and bladder, demonstrates appropriate hygiene), dressing (e.g., ties shoes, selects appropriate clothing), personal self-care (e.g., brushes teeth, dries after bathing), and basic domestic skills (e.g., clears table, puts belongings in proper place). The Community Living Skills subscales examine higher level skills involving time and punctuality (e.g., looks at a clock when it is "time" to do something, locates day and month on a calendar), understanding of money and value (e.g., counts, discriminates coins), basic work skills (e.g., indicates when a chore/task is finished, requests materials when needed), and 
home and community orientation skills (e.g., finds toys/objects in the home, stays in yard without wandering, crosses nearby streets alone). Validity studies of the SIB-R that focused on correlations with other tests of adaptive behavior have shown correlation coefficients ranging from .66 to .81 . The reliability of the SIB-R was found to range from .95 to .98 [30].

\section{RESULTS}

\section{A. Adaptive Profile}

To identify profiles of adaptive behavior in the 100 participants with WS, we compared the SIB-R's four domains in a repeated measures analysis of variance. A repeated measures ANOVA with a Greenhouse-Geisser correction determined that the mean cluster scores differed statistically significantly between four domains $(F(2.071$, $200.881)=42.663, P<0.001)$. Post hoc tests using the Bonferroni correction revealed a significant, relative strength in the Social Interaction and Communication Skills domain (Mean=66.94; SD=17.82 ), followed by the Personal Living Skills domain (Mean=58.37; $\mathrm{SD}=17.2$ ). The results also indicated relative weakness in Motor Skills domain (Mean=52.69; SD=19.77) and Community Living Skills domain (Mean=50.26; SD=19.65). However, there was no statistically significant difference in between the Motor Skills and Community Living Skills cluster scores.

TABLE I: CORRELATION COEFFICIENTS BETWEEN AGE AND SIB-R SCORES

\begin{tabular}{lcc}
\multicolumn{3}{c}{ FOR WS } \\
\hline \hline SIB-R Scores & Pearson $\mathrm{r}$ & Significance \\
Broad Independence Score & -.024 & .809 \\
Social Interaction and & $-.208^{*}$ & .038 \\
Communication & & \\
Personal Living & -.079 & .435 \\
Motor Skills & .057 & .574 \\
Community Living & -.010 & .924 \\
\hline \hline
\end{tabular}

*. Correlation is significant at the 0.05 level (2-tailed).

To further assess the relative weakness in the Community Living Skills domain, we compared age-equivalent scores from the subdomains in a repeated measure of analysis of variance, which was significant, $(F(1.081,107.029)=10.648$ $P=0.001)$. Post-hoc analyses revealed a relative strength in time (Mean age equivalent $=16.15$ years), and relative weakness in money, work, and home community orientation. Their age equivalent scores were 10.70, 10.34 and 11.01 years respectively. Since age equivalent scores for Motor Skills were not available, such analysis could not be performed for the Motor domain.

\section{B. Age-Related Changes in Adaptive Behavior}

The relation between adaptive behavior and chronological age (CA) was first assessed in a correlation between participants' CA and SIB-R broad independence and cluster scores (see Table I). SIB-R Social Interaction and Communication Skills score was the only cluster score that correlated significantly with age (negatively). In order to further examine age-related changes in adaptive profiles, participants were divided into three age groups. Group 1 consisted of 29 participants' ages' 12-20 years; Group 2 had 42 participants' ages' 21-35 years; Group 3 consisted of 27 participants' ages' 36-53 years. The mean ages, IQs, and SIB-R scores of these three groups were summarized in Table II. SIB-R Broad Independence scores were then correlated with chronological age in each of the three age groups. Although correlation in Group 1 ( $r=.27$, ns.) was higher than correlations in the other two groups $(r=-.04$, ns. and $r=.02$ for Group1 and 2 respectively), all three age groups showed a nonsignificant correlation between age and SIBR scores.

TABLE II: MEAN AGES, IQ, AND SIB-R SCORES BY AGE GROUPS (WITH STANDARD DEVIATION IN THE PARENTETHESE)

\begin{tabular}{llll}
\hline & \multicolumn{3}{c}{ Age Groups } \\
\cline { 2 - 4 } & $12-20(n=29)$ & $21-35(n=42)$ & $36-53(n=27)$ \\
\hline Age & $15.82(2.61)$ & $28.11(4.62)$ & $41.75(5.04)$ \\
IQ & $57.10(11.71)$ & $65.27(8.19)$ & $69.70(8.53)$ \\
Broad Independence & $48.55(20.83)$ & $49.14(19.79)$ & $46.37(19.25)$ \\
$\begin{array}{l}\text { Social Interaction } \\
\text { and Communication }\end{array}$ & $72.34(19.87)$ & $67.14(17.00)$ & $60.81(15.23)$ \\
Personal Living & $59.79(17.84)$ & $59.55(16.33)$ & $55.00(18.00)$ \\
Community Living & $49.72(19.13)$ & $50.83(20.41)$ & $49.93(19.72)$ \\
Motor Skills & $49.86(21.39)$ & $54.64(17.32)$ & $52.70(21.85)$ \\
\hline
\end{tabular}

To examine profiles with increasing CA, we further computed an analysis of variance comparing the age-equivalent scores on the three domains of the SIB-R (scores on the Motor domain is not available) across the three age groups. There was a statistically significant difference in SIBR age-equivalent scores based on age group status, Wilk's $=.695, F(6,188)=6.249, P<.001$, multivariate $\eta^{2}=.166$. Univariate results for this relationship demonstrated a significant effect for Personal Living Skills, $F(2,96)=$ $11.51, p<.001, \eta^{2}=.193$ and Community Living Skills, $F(2$, $96)=11.93, p<.001, \eta^{2}=.199$. For the Personal Living Skills, pairwise comparisons revealed statistically significant difference between group 1 (age equivalent=8.60) and group 2 (age equivalent=11.82) as well as between group 1 and group 3 (age equivalent=12.77). For the Community Living Skills, pairwise comparisons also indicated statistically significant difference between group 1 (age equivalent=8.48) and the other two groups (group 2 age equivalent=11.60; group 3 age equivalent=11.97). There is no significant age effect on the Social Interaction and Communication Skills and all three age groups had mean age equivalent scores for this domain ranging from 13.06 to 13.52 .

\section{DISCUSSION}

Present study examined the profiles and development of adaptive behavior in a wide age range of individuals with Williams Syndrome. The current findings were able to expand previous work by utilizing SIBR instead of VABS in order to assess adaptive motor functioning and community living skills in adolescents and adults with WS. Profile findings point to a strength in Social Communication and Interaction Skills and weaknesses in Motor Skills and Community Living Skills. Within the Community Living Skills, skills in the areas of money, basic work, and home/community orientation were relative weaknesses, 
whereas time skills appeared to be a relative strength. These results confirm other reports of adaptive profiles in individuals with WS. For example, Greer and colleagues [20] investigated the performance of children and adolescents with WS (ages 4 to 18 years) across different domains of adaptive behaviors using the VABS and found relative strengths in Socialization and Communication, while showing weakness in Daily Living. Mervis and colleagues [21] also examined adaptive behavior in children with WS aged from 4 to 8 years and the results showed that the domains of Socialization and Communication were relative strengths while Daily Living and Motor domains were areas of relative weakness.

In addition, the current study also extends previous observations in several ways. For example, we found that for adolescents and adults with WS, similar to their younger counterparts, relative weakness in the motor domain is also presented. Furthermore, the results indicated relative weakness in the community living skills domain. This is consistent with other research findings indicating that adults with WS have lower employment attainment rate. For example, Davies and colleagues [18] described in their study the adjustment of 70 adults with WS, in terms of self-help skills, independence, and occupational levels. They found that, despite having a similar degree of general cognitive impairment, relatively few individuals with WS were able to attain a reasonable level of independence, or cope with the demands of employment when compared to groups of adults with other intellectually disabling genetic disorders (i.e., Down syndrome and Prader-Willi syndrome). Furthermore, a study on 239 adults with WS (mean CA $=30$ years) revealed that only $4.6 \%$ of adults (11 of 239) held part-time paid employment (non-sheltered) and only $1.6 \%$ of the participants (4 of 239) held a full-time job [31].

At the same time, current study also found age-related differences in adaptive behaviors in individuals with WS. We found that adolescents with WS continued gaining adaptive skills in Personal Living and Community Living skills. But adults with WS did not appear to show such increasing abilities, suggesting an age-related plateau in the development of adaptive behavior during the adulthood. On the other hand, the Social Interaction and Communication skills in individual of WS appeared to be diminishing with age as evidenced by the statistically significant negative correlation between age and cluster score of this SIB-R domain. Research study [15] has shown that when assessed with VABS the Communication scores for adults with WS were significantly lower than both Socialization and Daily Living scores. Maybe this relative weakness in Communication in adult population with WS is associated with the age-related decline in Social Interaction and Communication domain observed in the current study. However, this age-related decline contradicted the findings of previous studies. Elison and colleagues [22] examined age associated changes in a number of different domains of functioning in adults with WS, including physical health, educational and occupational levels, self-care and independence, social interactions, and behavioral difficulties. Their cross-sectional analyses revealed no age related differences in the areas of functioning investigated. However, in the longitudinal sample, significant improvements were reported in physical health, self-care and occupational skills. They reported that social skills and adaptive behaviors had generally improved while behavioral difficulties had declined for adults with WS.

\section{CONCLUSION}

Although future studies are needed that longitudinally document strength and weaknesses, changing profiles, and trajectories of development for individuals with WS and other disorders, these and other findings provide preliminary evidence that may lead to more etiology-based programs of intervention. In terms of clinical implications, this study suggests that motor skills and community living skills are relative weakness in adaptive behavior experienced by adolescents and adults with WS. Therefore, interventions targeting visual-motor or motor skills would be most helpful. Instructional protocols focus on improving community living, especially in the areas of work skills, money skills, and home/community orientation should be developed to optimize skill acquisition in these areas. This study also demonstrated that there might be age-related decline in the area of social interaction and communication skills in adults with WS. Future studies need to examine this area and test possible hypothesis. It is also vital for professionals to keep in mind this population's different adaptive needs at different life stages so education, care, and therapy can be planned effectively to help them reach their full potential.

\section{REFERENCES}

[1] U. Bellugi, A. Järvinen-Pasley, T. Doyle, J. Reilly, and J. Korenberg, "Affect, social behavior and brain in Williams Syndrome," Current Directions in Psychological Science, vol. 16, pp. 99-104, 2007.

[2] C. B. Mervis and A. E. John, "Cognitive and behavioral characteristics of children with Williams Syndrome: Implications for intervention approaches," American Journal of Medical Genetics, pp. 229-248, 2010 .

[3] K. D. Hudson and E. K. Farran, "Drawing the line: Drawing and construction strategies for simple and complex figures in Williams Syndrome and typical development," British Journal of Developmental Psychology, 2010.

[4] C. B. Mervis, "The Williams Syndrome cognitive profile: Strengths, weaknesses, and interrelations among auditory short term memory, language, and visuospatial constructive cognition," in E. Winograd, R. Fivush, W. Hirst, eds., Ecological Approaches to Cognition: Essays in Honor of Ulric Neisser, Mahwah, NI: Erlbaum, 1999, pp. 193-227.

[5] C. Nagai, T. Inui, and M. Iwata, "Fading-figure tracing in Williams Syndrome," Brain Cognition, vol. 75, pp. 10-17, 2011.

[6] D. Elliott, T. N. Welsh, J. Lyons, S. Hansen, and M. Wu, "The visual regulation of goal-directed reaching movements in adults with Williams Syndrome, Down Syndrome, and other developmental delays," Motor Control, vol. 10, pp. 34-54, 2006.

[7] T. F. Doyle, U. Bellugi, J. R. Korenberg, and J. Graham, “"Everybody in the world is my friend' hypersociability in young children with Williams Syndrome," American Journal of Medical Genetics, pp. 263-273, 2004.

[8] E. M. Dykens, “Anxiety, fears, and phobias in persons with Williams Syndrome," Developmental Neuropsychology, vol. 23, pp. 291-316, 2003.

[9] A. Järvinen-Pasley, R. Adolphs, A. Yam, K. J. Hill, M. Grichanik, J. Reilly, D. Mills, A. L. Reiss, J. R. Korenberg, and U. Bellugi, "Affiliative behavior in Williams Syndrome: Social perception and real-life social behavior," Neuropsychologia, vol. 48, pp. 2110-2119, 2010.

[10] H. Tager-Flusberg and K. Sullivan, "A componential view of theory of mind: Evidence from Williams Syndrome," Cognition, vol. 76, pp. 59-89, 2000. 
[11] U. Bellugi, E. Lichtenberger, W. Jones, Z. Lai, and M. S. George, “The neurocognitive profile of Williams Syndrome: A complex pattern of strengths and weaknesses," in U. Bellugi, M. S. George, eds., Journal of Cognitive Neuroscience (Special Issue), vol. 12, pp. 1-29, 2000.

[12] M. Liss, B. Harel, D. Fein, D. Allen, M. Dunn, C. Feinstein et al., "Predictors and correlates of adaptive functioning in children with developmental disorders," Journal of Autism and Developmental Disorders, vol. 31, pp. 219-230, 2001.

[13] P. Szatmari, S. E. Bryson, M. H. Boyle, D. L. Streiner, and E. Duku, "Predictors of outcome among high-functioning children with autism and asperger syndrome," Journal of Child Psychology and Psychiatry, vol. 44 , pp. 520-528, 2003.

[14] S. D. Nuovo and S. Buono, "Behavioral phenotypes of genetic syndromes with intellectual disability: Comparison of adaptive profiles," Psychiatry Research.

[15] P. Howlin, M. Davies, and O. Udwin, "Cognitive functioning in adults with Williams Syndrome," Journal of Child Psychology and Psychiatry, vol. 39, no. 2, pp. 183-189, 1998.

[16] C. B. Mervis and B. P. Klein-Tasman, "Williams Syndrome: Cognition, personality, and adaptive behavior," Mental Retardation and Developmental Disabilities Research Reviews, vol. 6, pp. 148-158, 2000.

[17] A. Gosch and R. Pankau, "Social-emotional and behavioral adjustment in children with Williams-Beuren syndrome," American Journal of Medical Genetics, vol. 53, pp. 335-339, 1994.

[18] M. Davies, P. Howlin, and O. Udwin, "Independence and adaptive behavior in adults with Williams Syndrome," American Journal of Medical Genetics, vol. 70, pp. 188-195, 1997.

[19] S. S. Sparrow, D. A. Balla, and D. V. Cicchetti, Vineland Adaptive Behavior Scales-Interview Edition, Circle Pines, MN: American Guidance Service, 1984.

[20] M. K. Greer, F. R. Brown, G. S. Pai, S. H. Choudry, and A. J. Klein, "Cognitive, adaptive, and behavioral characteristics of Williams Syndrome," American Journal of Medical Genetics, vol. 74, pp. 521-525, 1997

[21] C. B. Mervis, B. P. Klein-Tasman, and M. E. Mastin, "Adaptive behavior of 4-through 8-year-old children with Williams Syndrome," American Journal on Mental Retardation, vol. 106, pp. 82-93, 2001.

[22] S. Elison, C. Stinton, and P. Howlin, "Health and social outcomes in adults with Williams Syndrome: Findings from cross-sectional and longitudinal cohorts," Research in Developmental Disabilities, vol. 31, pp. $587-599,2010$
[23] R. W. Woodcock and M. B. Johnson, "Woodcock-Johnson tests of achievement," DLM Teaching Resources, 1989.

[24] M. Porter and H. Dodd, "A longitudinal study of cognitive abilities in Williams syndrome," Developmental Neuropsychology, vol. 36, pp. 255-272, 2011.

[25] J. Carr, "Long term outcome for people with Down's syndrome," Journal of Child Psychology and Psychiatry, vol. 35, pp. 425-439, 1994.

[26] Y. M. Searcy, A. J. Lincoln, F. E. Rose, E. S. Klima, N. Bavar, and J. R. Korenberg, "The relationship between age and IQ in adults with Williams syndrome," American Journal of Mental Retardation, vol. 109, pp. 231-236, 2004.

[27] O. Udwin, H. Davis, and P. Howlin, "A longitudinal study of cognitive abilities and educational attainment in Williams syndrome," Developmental Medicine and Child Neurology, vol. 38, pp. 1020-1029, 1996.

[28] D. Elliott and L. Bunn, "Motor disorders in children with intellectual disabilities," in D. Dewey and D. E. Tupper (Ed.), Developmental Motor Disorders: A Neuropsychological Perspective, New York: Guilford Press, 2004, pp. 137-151.

[29] C. Lebel, L. Walker, A. Leemans, L. Phillips, and C. Beaulieu, "Microstructural maturation of the human brain from childhood to adulthood," NeuroImage, vol. 40, pp. 1044-1055, 2008.

[30] R. H. Bruininks, R. W. Woodcock, R. F. Weatherman, and B. K. Hill, Scales of Independent Behavior-Revised, Chicago, IL: Riverside, 1996.

[31] P. Howlin and O. Udwin, "Outcome in adult life for people with Williams syndrome: Results from a survey of 239 families," Journal of Intellectual Disability Research, vol. 50, pp. 151-160, 2006.

Trista Juhsin Fu was born and raised in Taiwan. She obtained a bachelor degree in education from National Chengchi University in Taipei, Taiwan in 1998. She later studied in the U.S. and received a master degree in counseling from University of Wisconsin-Madison, and a Ph.D. degree in clinical psychology from California School of Professional psychology at Alliant International University in San Diego, California.

She is currently an assistant professor in College of Education at National Chengchi University. Dr. Fu completed an APA predoctoral psychology fellowship with School of Medicine at Yale University. Her research interests include mental health in individuals with neurodevelopmental disorders, couple and family therapy, and parenting-related issues.

Dr. Fu is a member of the American Psychological Association and Asian American Psychological Association. 